\title{
XIII. The anomalous dispersion, absorption, and surface-colour of nitroso-dimethyl-aniline, with a note on the dispersion of toluine
}

\section{R.W. Wood}

To cite this article: R.W. Wood (1903) XIII. The anomalous dispersion, absorption, and surfacecolour of nitroso-dimethyl-aniline, with a note on the dispersion of toluine, Philosophical Magazine Series 6, 6:31, 96-112, DOI: 10.1080/14786440309462994

To link to this article: http://dx.doi.org/10.1080/14786440309462994

曲 Published online: 15 Apr 2009.

Submit your article to this journal

Џll Article views: 2

Q View related articles $\longleftarrow$

Citing articles: 2 View citing articles ᄃ 
In air at atmospheric pressure the falling off of the conductivity with time is much slower than at low pressures. Thus Mr. H. A. Wilson* found the following numbers for the current in amperes between two coaxial platinum cylinders at the temperatures stated. The potent:al-difference was 800 volts and the outer tube was negative.

\begin{tabular}{|c|c|c|c|}
\hline Temperature $800^{\circ}$. & $900^{\circ}$ & $1000^{\circ}$. & $1100^{\circ}$. \\
\hline July $6 \ldots \ldots \ldots 8 \times 10-6$ & $40 \times 10^{-6}$ & $120 \times 10-6$ & $400 \times 10-6$ \\
\hline July $10 \ldots \ldots \ldots 2 \times$, & $11 \times n$ & $50 \times$, & $140 \times \quad "$ \\
\hline July $39 \ldots \ldots \ldots \cdot 1 \times$," & $7 x$ & $2 \cdot 5 x$ & $8 x$ \\
\hline
\end{tabular}

The greater persistence of the positive leak at high pressures is probably to be attributed either to the greater difficulty experienced by the induced activity in ditfusing away, or by the fact that a new active layer is continually being reformed on the wire. Which of these two views is correct is a matter for future experiment to decide. In the present experiments the induced activity appeared to be more persistent at $3 \mathrm{~mm}$. than at $06 \mathrm{~mm}$.

The activity induced by the luminous discharge, and several other points which are not definitely settled in this communication, are being further investigated at present.

The experiments were carried out in the Cavendish Laboratory, and the author's best thanks are due to Professor J. J. Thomson for his kindly interest and advice during the course of the work.

XIII. The Anomalous Dispersion, Absorption, and SurfaceColour of Nitroso-dimetleyl-aniline, with a Note on the Dispersion of Toluine. By R. W. Woon, Professor of Experimental Plysics in the Johns Hopkins University $\dagger$.

[Plates VI.-VIII.]

THEE very high dispersion of the aniline dyes and other absorbing modia is due, as is well known, to the fact that the absorption-band lies within the visible spectrum. The absorbing power of these substances, for wave-lengths far removed from the centre of the absorption-band, is so great that only prisms of very small angle can be used, which puts a limit on the length of the spectrum which can be obtained with them.

* Phil. Trans. vol. cxcvii. p. 415.

+ Communicnted by the Author. 
In the case of the so-called transparent substances, the absorption-band lies so far down in the ultra-violet that the steepness of the dispersion-curve in the visible spectrum is not comparable with that of the substances which are said to exhibit anomalous dispersion. If, however, we push the curve down to the absorption-band, employing some photographic method, we find that it may be even steeper than the curve for cyanine in the red and orange.

I have found that nitroso-dimethyl-aniline is of peculiar interest, in that it fills in the gap existing between the aniline dyes and ordinary transparent substances. It has a band of metallic absorption in the violet, and is at the same time fairly transparent to the red, yellow, and green. The substance melts at $85^{\circ} \mathrm{C}$., and can be formed into prisms between small strips of thin plate-glass. The strips should be about $2 \mathrm{cms}$. long, and are best fastened together with one of the small clamps used with rubber tubing. It is best to melt the material on the end of one of the strips, the other being warmed over the same flame, and then clamp the two together with a piece of a match between the other ends, to give the required prismatic form. A candle-flame viewed through the prism is spread out into a most remarkable spectrum fifteen or twenty times as long as one given by a glass prism of the same angle. It is instructive to have a prism of the same angle made of Canada balsam or some such substance pressed out between two similar glass strips.

In addition to its remarkable dispersion, the nitroso, as I shall call it for short, exhibits a most beautiful violet surfacecolour, which can best be exhibited by employing a small cell heated by steam such as I shall describe later on.

The substance possesses in addition several other interesting features. It has, for example, in addition to its sharp and narrow band of metallic absorption in the violet, a weaker pair of bands near the end of the ultra-violet which flatten ont the dispersion-curve, but do not bend it into oppositely directed branches as the stronger band does. Moreover, the substance can be vaporized without decomposition, which makes it possible to compare its optical properties in the three states-solid, liquid, and gaseous.

I shall take up in order the dispersion in the visible spectrum, the ultra-violet dispersion, the reflecting power in different parts of the spectrum, the angles of maximum polarization, and the changes in the position of the absorptionband which accompany a change of state. 'The various results will finally be discussed in their bearing on the electromagnetic theory of dispersion and absorption.

$$
\text { Plil. Mag. S. 6. Vol. 6. No. 31. July } 1903 .
$$




\section{Dispersion in the Visible Spectrum.}

As the refractive index of the nitroso changes very rapidly with the temperature, it was necessary, in making the determinations of the dispersion, to keep the temperature of the melted substance eonstant. The point chosen was the solidifying point, as it simplified the experimental conditions. The prism was constructed of a pair of interferometer plates accurately plane-parallel, and was mounted on the table of a spectrometer in a small clamp-frame made especially for it. A current of hot air was directed against the prism by means of a bent glass tube, under one end of which a small gasflame was burning. The slit of the spectrometer was illuminated with approximately monochromatic light furnished by a monochromatic illuminator built by Fuess. This extremely useful instrument is not as well known as it deserves to be, and a few words regarding it may not be out of place. It is essentially a small spectroscope with collimator and telescope at right angles. The two prisms, which are inclosed in the hody of the instrument, are turned by means of a micrometer screw, from the reading of which the wave-length can at once be determined from the calibration-curve of the instrument. The eyepiece can be removed, and a draw-tube carrying an adjustable slit inserted in its place. The spectrum can bo made to pass across this slit by turning the micrometer screw, and by noting the readings when known lines in the spectrum fall on the slit, the instrument can be calibrated. 'This is easily and quickly accomplished by means of an ingeniously arranged microseope which can be thrown into and out of position as desired. A small lens carried in a tube in tront of the slit focusses the monochromatic light which issues from it on the slit of the spectrometer.

The dispersion of the nitroso was measured in the following manner :-The prism having been set at minimum deviation, and the usual adjustments made, the deviated image of the slit, illuminated in monochromatic light, was brought into the field of the telescope. The gas-flame was then moved out of position and the current of hot air stopped. As the fluid nitroso cooled, the deviation increased, the reading being taken just at the point of solidification, when the image disappeared gradually owing to the crystallization of the medium. The warm air was then turned on again, and a second reading taken in the same way. A number of prisms were used, the angles varying from 1 to 10 degrees, those of small angle being necessary when working with the blnish-green, owing to the absorption. In this way the dispersion was 
measured between the extreme red and wave-length $\cdot 00050$, below which point the prims refused to transmit sufficient light to make readings possible. The values obtained in this way are given in the following table.

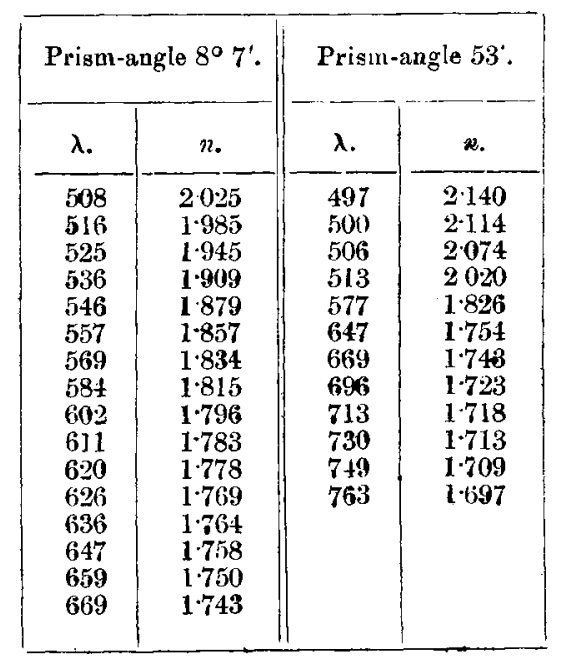

The results are shown graphically in Plate VI., together with the dispersion-curve for bisulphide of carbon for the same region of the spectrum. The remarkable dispersive power of the nitroso is at once apparent when we compare the two curves, and contrast it with that of the bisulphide, which has the highest dispersive power of any substance in common use.

Over the region of the spectrum given above, the nitroso can be considered as a transparent substance, and the dispersion formula for transparent substances can be applied to it. From three values of $n$ and the corresponding values of $\lambda$ the value of $\lambda^{\prime}$ the centre of the absorption-band can be calculated.

In the case of selenium, which has a dispersion-curve resembling that of the nitroso, the absorption appears to increase steadily from the yellow down to the extreme ultraviolet, making it impossible to determine experimentally the centre of the absorption-band which is chiefly responsible for the dispersion. Applying the formula for transparent substances to the values found for selenium, I found that if we assume a single absorption-band, its centre must be at wavelength $\cdot 00056$. To account for the continued absorption as $\mathrm{H} 2$ 
we pass down the spectrum, we have only to assume that there is a series of bands of which the calculated one is the first member. I expected that the nitroso would behave in a similar manner, but found on examining its transmission that the absorption, which commenced at $\lambda=\cdot 0005$ ended quite abruptly at about $\cdot 00037$, the substance transmitting the ultra-violet almost down to the last cadmium lines. This property of the substance enabled me to prepare screens transparent only to ultra-violet light, which I bave described in a previous paper (Phil. Mag. Feb. 1903).

The transparency of the nitroso on the ultra-violet side of the absorption-band is, however, very much less than on the green side, as I soon found in endearouring to measure the dispersion by crossing a prism of the substance formed between quartz plates, with the prism of a quartz spectrograph. No trace of the spectrum on the more refrangible side of the absorption-band appeared on the photographic plate. After a number of failures, which obviously resulted from the insufficient transparency of the nitroso prism, combined with the necessarily short exposure, I abandoned this method, and made some rough determinations of the ultraviolet dispersion by observations on the angle of maximum polarization for these wave-lengths. The results, while not very accurate, gave unmistakable evidence of anomalous dispersion, the refractive index for wave-lengths below the absorption-band ranging from $1 \cdot 1$ to about $1 \cdot 5$. I was subsequently able to use the method of crossed prisms, by employing very acute prisms, kept warm by means of an electrically heated platinum wire, which made long exposures possible.

I shall first, however, consider the polarization phenomena accompanying the reflexion of light from the surface of the fluid nitroso. The substance was kept in a liquid condition by means of a small cell heated by a current of steam (fig. 1, p. 101).

On examining the light reflected at a fairly large angle with a Nicol prism, it will be found to vary from light blue to deep violet and purple, as the angle of incidence is increased, the nicol being held in such a position as to refuse transmission to the light polarized by reflexion. If a spectroscope is placed behind the nicol, a dark band will be seen crossing the spectrum, which shifts its position as the incidence angle varies. 'The centre of this dark band is evidently the wave-length for which the angle of incidence happens to be the angle of maximum polarization; or, in other words, the refractive index of the substance for this wave-length is the tangent of the angle of index. In the case of glass and 
substances of low dispersion, the different colours are polarized at nearly the same angle, i. $e$. very little colour-effect is observed when the reflected light is examined with the nicol.

Fig. 1.
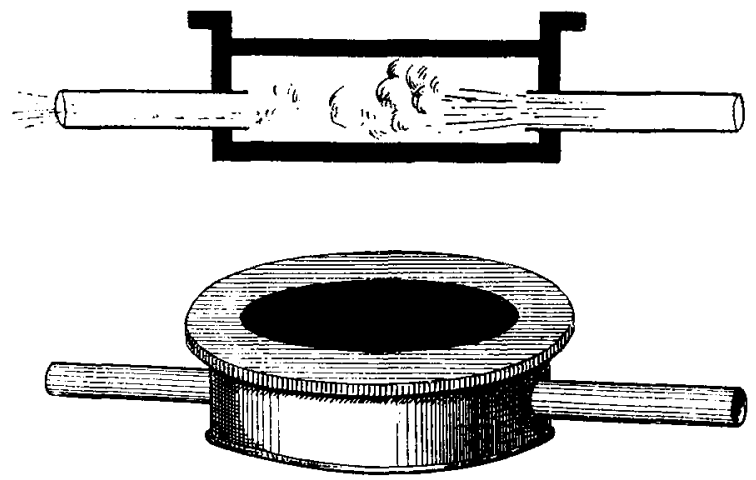

In these cases the dark band to which I have referred is so broad as to occupy practically the entire visible spectrum. In the case of a substance with as high a dispersion as that of the nitroso, the angle of maximum polarization is quite different for the different colours, so that the dispersion may be determined by observing the position of the centre of the dark band and the angle of incidence. It was found that values agreeing very closely with those obtained with the prisins could be obtained by this method in the yellow, green, and greenish-blue portions of the spectrum. On attempting to drive the band through the orange and into the red, it broadened so much that it was quite impossible to locate its centre with any precision. This was of course due to the fact that the dispersion in the red and orange is not sufficient to make the method very accurate in this part of the spectrum. The dispersion of selenium was also determined in this manner, and found to agree very well with the results obtained with prisms. To apply the method to the ultra-violet the observations were made photographically with a small quartz spectrograph made by Fuess. This instrument was furnished with a Rochon prism mounted immediately behind the quartz collimation-lens. This prism, as furnished by the maker of the instrument, refused to transmit the ultra-violet, and I found that the two balves had been cemented together with balsam, which I replaced with glycerine. Some preliminary experiments were made with selenium mirrors, as they were easier to handle than the fluid cell. The light from a cadmium 
spark was reflected into the slit of the instrument at various angles of incidence, and dark bands were found running across the two polarized spectra furnished by the Rochon prism. I spent some time endeavouring to extract data from these bands, and finally came to the conclusion that either they were not due to the seleetive polarization by reflexion, or else that the dispersion-curve as determined for selenium by means of the interferometer was in error. On experimenting further, I found that these bands were due solely to the rotatory dispersion of the light in the quartz collimatinglens, some colours being rotated through such an angle as to be quenched in one spectrum by the Rochon, and others in such a degree as to be absent in the other. I mention this defect in the instrument, as it may be of interest to others working along similar lines. The proper design of the instrument should have called for a collimating-lens made of two thin lenses, one of right-handed, the other of left-handed quartz. I remedied the defect in my instrument by placing a plate of left-handed quartz immediately behind the lens. This plate had a thickness equal to the thickness of the lens at its centre, and abolished the bands entirely, when the lens was stopued down to a small area at the centre.

I found, however, in working with the horizontal cell of fluid nitroso, that better results were obtained by using a Nicol prism in front of the slit of the spectrograph than with the Rochon prism. The nicol was made transparent to ultraviolet light by separating the two halves, cleaning off the balsam, and substituting glycerine.

Evidence of the very low value of the refractive index on the ultra-violet side of the absorption-band was obtained before any polarization experiments were tried. The light of a cadmium spark was reflected from the pool of liquid nitroso at nearly normal incidence, and then thrown into the spectrograph by means of a quartz total-reflecting prism. A series of spectra was taken, with times of exposure rarying from 2 seconds to 3 minutes. By comparison of the different spectra it was possible to form a rough estimate of the reflecting power in different parts of the spectrum. A similar series was made with a flat reflector of magnalium, which is remarkably constant in reflecting power throughout the entire spectrum. It was found that the nitroso, in the region of its absorption-band, reflected almost as strongly as magnalium, while just on the ultra-violet side of the band there was a gap in the spectrum, due to the very low reflecting power which the substance has for these waves (Plate VIII. tig. 8). The reflexion coefficient at this point was estimated at about 
2 per cent. only, while a short distance further up the spectrum the coefficient is at least 80 per cent.* This indicates that the refractive index has a very low value at the point of minimum reflexion. The following table gives an idea of the reflecting power of the nitroso in the various regions of the spectrum as compared with the magnalium. The values are of course only approximate, being deduced by picking out two spectra (one from nitroso, the other from magnalium) which showed the same intensity at the given wave-length, and then comparing the times of exposure. The reflecting power is expressed as a fractional part of the reflecting poner of magnalium.

\begin{tabular}{cc|cc}
$\lambda$. & Refl. Power. & $\lambda$. & Refl. Power. \\
217 & $1 / 2$ & 380 & $1 / 2$ \\
240 & $1 / 2$ & 399 & 1 \\
290 & $1 / 3$ & 467 & 1 \\
330 & $1 / 6$ & 508 & $1 / 2$ \\
343 & $1 / 12$ & 535 & $1 / 4$ \\
350 & $1 / 40$ & 569 &
\end{tabular}

When the incidence angle is increased and the reflected light examined with a Nicol prism, a most beautiful surfacecolour appeared, ranging from a brilliant blue through violet to a reddish purple. This is very easy to understand. The violet light within the region occupied by the absorptionband is metallically reflected at all angles of incidence, consequently it is always present in excess in the reflected light, and is never cut off by the nicol. As the angle of incidence is increased, the polarizing angle for red light is first reached, and the red end of the spectrum disappears, being cut off by the nicol, the reflected light appearing blue. As the angle increases more of the spectrum is removed by the nicol, and the colour changes to deep violet, the dark band advancing down the spectrum. Finally, by further increase the red appears again in full intensity, being reflected unpolarized, and mixing with the metallically reflected violet gives the brilliant reddish purple. Very similar appearances can be observed with selenium, though the colours are not as saturated and not nearly so brilliant.

Having roughed out, so to speak, the dispersion in the ultra-violet by photographing the spectrum of the reflected

* This value is much too high. It was based on the supposition that the magnalium reflected 80 per cent., which was subsequently found to be incorrect, the metal containing too little marnesium, and having a rather low refecting power. 
light at various angles of incidence through a Nicol prism, an attempt was next made to secure more reliable data by means of the method of crossed prisms. A prism of small angle, held in a clamp, was mounted with its refracting edge in a horizontal position, immediately behind the prism of the quartz spectrograph. A fine platinum wire immediately below the edge of the prism, when heated by a current of suitable strength, kept the nitroso in a fluid condition. A system of small screens was arranged so that the light could either be made to pass through the nitroso prism, or through a small clear space in the quartz plates immediately above it, thus photographing the deviated and undeviated spectra one above the other. The current strength was so adjusted as to keep the temperature of the prism as nearly as possible at the melting-point of the nitroso. It was possible in this way to give exposures of an hour or more, and obtain a photographic record of the dispersion-curve from the orange down to the extreme end of the ultra-violet. The cadmium spark was employed as a source of light, and the slit of the spectrograph was diaphragmed down to a length of less than a millimetre in order to obtain very narrow spectra. As the conditions necessary for success were determined by repeated experiments, prisms of larger angle were used, and the two spectra, which overlapped in the first experiments in the region where the refractive index had a low value, were completely separated. I found that a great improvement resulted from carefully grinding the edge of the quartz plate, which rests against the other plate, perfectly straight with fine emery. It was not until this expedient was adopted that the larger angles became possible. The deviated spectrum is quite sharp except close to the edge of the absorption-band, where absorption produces a broadening of the image by reducing the effective width of the beam of light, as is always the case with strongly absorbing prisms. It is even possible to follow the general trend of tbe dispersion-curve right through the band of metallic absorption, though the broadening resulting from diffraction is, in this region, so great that accurate measurements were impossible. The continuity of the curre is shown, however, better than I have ever seen it in any photograph. To deduce numerical values from the photographs, the distances between the spectra were measured with i dividing-engine at the principal cadmium lines. From these distances the actual angular deviations were calculated, making proper allowance for the fact that the plate stood at an angle-i.e., the focal length of the lens of the spectrograph was very different in the different regions of the spectrum. 
The angle of the prism was calculated from the deviations in the yellow and green, for which region the refractive index had been already determined with considerable accuracy. In spite of the rather crude method, the results obtained with the different prisms agreed surprisingly well.

It will be seen from the photographs, some of which are reproduced in fig. 3, Plate VIII., that the deviated and undeviated spectra come together at the ultra-violet edge of the principal absorption-band, indicating a refractive index not much above unity.

(a) deviated spectrum obtained with prism of very small angle; $(b)(c)(d)$ deviated and undeviated spectra obtained with prisms of larger angle. The continuity of the curve through the absorption-band can be seen in $(a)$ and $(b)$, also in $(e)$, which was enlarged from $(b)$. The ultra-violet is to the left in the first four of these figures.

'The prism-angles in these experiments varied from $20^{\prime}$ to $2^{\circ}$.

It was found possible to get a fairly accurate determination of the refractive index for wave-length 48 from these photographs, the mean of several determinations giving $2 \cdot 28$ as the value. The values found on the ultra-violet side of the band are given in the form of a curve in Plate VII. This portion of the curve is especially interesting, as the effect of the ultra-violet absorption-bands is most strikingly shown. The weak band at wave-length 25 causes a dip in the curve, which has a maximum at $\lambda=28.5$, after which it again rises rapidly as it approaches the heavier absorption-band situated not far from $\lambda=20$.

On this same plate I have given the dispersion in the visible spectrum, together with the value $n=8 \cdot 85$ for wavelength $\lambda=43$ the centre of the absorption-band, which was determined from the constants of elliptical polarization. The trend of the curve within the absorption-band I have sketched in free-hand, as well as the general form and position of the absorption-bands.

I bave already shown that the reflecting power of the nitroso for the wave-lengths immediately adjoining the absorption-band on the ultra-violet side is exceedingly small, which is not surprising when we consider that the refractive index for this region is not very different from air. If now we consider a quartz-nitroso surface instead of an air-nitroso surface, we should expect fairly strong reflexion at this point, the nitroso acting as the rarer medium, while further down the spectrum, at about wave-length 29 , where the indices of the quartz and nitroso are very nearly the same, we should expect scarcely any reflexion at all. 
This was found to be the case. An acute prism of quartz was ground and polished, and one face of it brought in contact with a small drop of nitroso fused on a strip of blackened brass. One end of the strip was lueated in a flame, the nitroso remaining fluid as a result of the heat conduction along the strip. The object of using the blackened brass was to get rid of reflexion from the back surface of the nitroso. The light reflected from the front surface of the quartz was thrown to one side, owing to the inclination, so that everything was eliminated except the reflexion from the quart\%nitroso surface. The spectrum of this selectively reflected light is shown in fig. 5 (Plate VIII.). The minimum at wavelength 36 will be seen to have disappeared, and a new one will be found at 29 just about where we should expect it. This minimum would doubtless be more pronounced were it not for the fact that the absorption coefficient has at this point a not inconsiderable value, which will cause the bounding surface in question to have a higher reflecting power than if both media were perfectly transparent.

\section{Absorption of Solid, Liquid, and Gaseous Nitroso.}

No determinations of the dispersion of the solid nitroso have been made, owing to the difficulty of getting suitable prisms: when the fluid prisms cool off the nitroso crystallizes in aborescent forms, and no longer transmits regularly. If the prism is held close to the eye, and a lamp-flame viewed through it, enormously deviated spectra are seen, due to the formation of crystals having a much larger angle than the prism. The substance in the solid condition is doublerefracting, the two spectra which every prism furnishes being extinguished in turn by a revolving Nicol prism. The absorption-band of the solid nitroso was studied by photography. A drop of fluid nitroso was pressed in a clamp betreen two hot plates of quartz, which were allowed to cool under pressure. In this way a very thin film of the solid substance was obtained. On photographing a spectrum through this screen, it was found that the principal absorption-band had broken up into two placed symmetrically with respect to the band shown by the fluid. One of these doubtless belongs to the ordinary, the other to the extraordinary ray. The centres of the bands are at wave-lengths 36 and 46 , while the centre of the single band possessed by the liquid is at $\lambda=43$.

In fig. 6 (Plate VIII.) we have two photographs of this double band.

The absorption of the liquid nitroso does not differ very materially from that of its solution in glycerine, which $I$ 
have described in a previous paper on screens transparent only to ultra-violet light *.

Th absorption-spectrum was photographed by pressing out a drop of the fluid substance between a quartz lens and plate, the whole being kept warm by a current of hot air. In this way a film of variable thickness was obtained which, when brought close to the slit of the quartz spectrograph, enabled a record to be made of the relative intensities, positions, and general form of the absorption-bands. One of these photographs is shown in fig. 7, Pl. VIII.

The nitroso begins to vaporize at a temperature only a little above its melting-point, and the absorption of the vapour is interesting, for unlike most absorbing vapours and gases this substance shows a broad absorption-band, similar to the liquid only shifted well down into the vltra-violet. The nitruso was vaporized in a glass tube, the ends of which were closed with quartz plates. The tube was previously exhausted, and was heated by a water-bath through which it passed. The absorption-spectrum was photographed at different temperatures ranging from $85^{\circ}$, the melting-point, to $100^{\circ}$. The absorption does not begin as a narrow line, as is usually the case with gases, but with a broad band, which increases in intensity as the density of the vapour increases. The centre of this band is at wave-length 34 , while the centre of the band in the case of the liquid is at 43 .

I made numerous experiments to see whether the density of the vapour could be sufficiently increased to cause a shift in the position of the band towards the red, all of which failed owing to the fact that the nitroso decomposes at temperatures above $150^{\circ}$. The substance was heated in strong sealed bulbs in an air-bath, but decomposition always resulted before a density sufficient to shift the absorption-band in a measurable degree had been obtained.

On the other hand, the position of the absorption-band can be shifted by increasing the density of the medium in which the nitroso-vapour is present. If a solution of nitroso in ether is heated above its critical temperature in sealed glass tubes, the centre of the absorption-band can be given almost any position between that of the vapour-band and the solutionband, by varying the amount of ether in the tabe, or, in other words, by varying the density of the vapour.

I made a number of attempts to prove that the double overlapping band sbown by the solid was connected with the double refraction of the nitroso crystals, by placing a nicol before the slit of the spectrograph and photographing the 
absorption-spectrum of a thin crystalline film of the substance between two quartz plates. A number of spectra were taken with the nicol in different positions, a comparison of which gave unmistakable evidence that one band belonged to the ordinary, the other to the extraordinary ray. In no ease did either of the bands disappear entirely, owing to the fact that they overlap, which makes it impossible to have completo transparency with the nicol in any position. No measurements have as yet been made of the values of the extinctioncoefficient in different parts of the spectrum, owing to the difficulty of preparing a film thin enough to transmit light within the absorption-band. Though it is possible to get a wedge-shaped film between a lens and a flat plate which transmits all wave-lengths to a certain degree in its thinnest portions, a film of this nature is quite unsuitable for quantitative measurements of the absorption. Probably by working with solutions in glycerine of different concentrations and thicknesses, a fairly correct idea of the absorption-curve could be obtained by calculation, though this method would be open to some objections. Until the substance has been investigated in the infra-red, and until the dielectric constant has been determined, the dispersion-formula cannot be applied to it to the best advantage. At the present time, ficilities for investigating these two points are not at my disposal, but I expect in the near future to investigate them. In the meantime it is instructive to apply the formula to the results which have already been obtained.

\section{The Dispersion Formula.}

For parts of the spectrum in which the extinction-coefficient has a small value, the dispersion is represented by the formula

$$
n^{2}=1+\Sigma \frac{m_{k} \lambda^{2}}{\lambda^{2}-\overline{\lambda_{k}}}
$$

in which $\lambda_{k}$ are the wave-lengths of the centres of the absorption-bands (nearly), and $m_{k}$ constants for these bands, the summation being taken for all of the absorption-bands, whether near or far removed from the portion of the spectrum under consideration. If the bands are far removed from this region the fraction is practically equal to unity, and the constants $m^{\prime}, m^{\prime \prime}$, \&c. have merely to be added or subtracted according to whether they lie on the more refrangible or less refrangible side of the spectral region under investigation, i. e. according as the sign of $\frac{\lambda^{2}}{\lambda^{z}-\lambda^{\prime 2}}$ is positive or negative. 
and Surface-Colour of Nitroso-dimethyl-aniline.

For a medium having but a single absorption-band, the formula takes the form

$$
n^{2}=1+\frac{m^{\prime} \lambda^{2}}{\lambda^{2}-\lambda^{\prime 2}}
$$

This formula can, I think, be applied to sodium vapour, judging from results recently obtained. Most other substances have, however, a second band further along in the ultraviolet, though for the region investigated experimentally this is so far removed that $m^{\prime \prime}$ can be considered constant. The formula then takes the form

$$
n^{2}=m+\frac{m^{\prime} \lambda^{2}}{\lambda^{2}-\lambda^{\prime 2}},
$$

in which $m=m^{\prime \prime}+1$. This formula has been found to represent the dispersion of most transparent substances.

I have applied this formula to the region of the spectrum for which the nitroso is most transparent, using the data obtained with the spectrometer and prisms of comparatively large angle.

The position of the centre of the absorption-band can be calculated from three observed values of the refractive index $n_{1}, n_{2}, n_{3}$ for wave-lengths $\lambda_{1}, \lambda_{2}, \lambda_{3}$ from the formula

in which

$$
\lambda^{\prime 2}=\frac{\lambda_{3}{ }^{2}\left(\lambda_{1}{ }^{2}-\lambda_{2}{ }^{2}\right)-\lambda_{2}{ }^{2}\left(\lambda_{1}{ }^{2}-\lambda_{3}{ }^{2}\right) \mathrm{C}}{\left(\lambda_{1}^{2}-\lambda_{2}{ }^{2}\right)-\left(\lambda_{1}{ }^{2}-\lambda_{3}{ }^{2}\right) \mathrm{C}},
$$

$$
\mathrm{C}=\frac{n_{2}^{2}-n_{1}^{2}}{n_{3}^{2}-n_{1}{ }^{2}}
$$

This formula gives $\lambda^{\prime}=43 \cdot 1$ for the centre of the absorptionband, a value agreeing closely with the observed value. Further,

$$
\begin{aligned}
m^{\prime} & =\frac{\left(n_{3}{ }^{2}-n_{1}{ }^{2}\right)\left(\lambda_{1}{ }^{2}-\lambda^{\prime 2}\right)\left(\lambda_{3}{ }^{2}-\lambda^{\prime 2}\right)}{\lambda^{\prime 2}\left(\lambda_{1}{ }^{2}-\lambda_{3}{ }^{2}\right)} \\
m & =n_{2}{ }^{2}-\frac{m^{\prime} \lambda_{2}{ }^{2}}{\lambda_{2}{ }^{2}-\lambda^{\prime 2}} .
\end{aligned}
$$

The values found for $m^{\prime}$ vary slightly with the region of the spectrum in which the values of $n$ and $\lambda$ are chosen. This indicates the presence of one or more absorption-bands in the infra-red. The mean values found were

$$
\begin{aligned}
& m^{\prime}=0 \cdot 53 \\
& m=2 \cdot 13 .
\end{aligned}
$$

This should make the dielectric constint $2 \cdot 66$, if no infra-red 
absorption-bands are present. If it had turned out that $m$ equalled unity, the inference would have been that the absorption-band iat $\lambda=43$ was the only one present. The large value of $(m-1)$ indicates that there are bands of metallic absorption in the ultra-violet. As I have already shown, a strong absorption-band exists a little below $\lambda=20$, and a weak one at $\lambda=25$.

Though the formula as it stands represents fairly well the dispersion in the red, yellow, and green, it breaks down if we try to apply it to the values found in the ultra-violet by the photographic application of the method of crossed prisms. This is due to the fact that we are getting into the region in which $\frac{\lambda^{z}}{\lambda^{2}-\lambda^{\prime 2}}$ is no longer approximately equal to unity.

This quantity increases in magnitude as $\lambda$ decreases, and the values of $n$ will consequently be higher than those calculated on the assumption that the quantity is equal to unity. To meet this contingency we must use the formula (neglecting the weak band at $\lambda=25$ )

$$
n^{2}=1+\frac{m^{\prime} \lambda^{2}}{\left(\lambda^{2}-\lambda^{\prime 2}\right)}+\frac{m^{\prime \prime} \lambda^{2}}{\lambda^{2}-\lambda^{1 / 2}}
$$

Since $m=2 \cdot 13=m^{\prime \prime}+1$, we can take $m^{\prime \prime}=1 \cdot 13$.

The value of the refractive index $n$ for $\lambda=34$, calculated from the original or simplified formula, is $n=1 \cdot 1$, while the obscrved value is 1.3 ; in other words, the value is raised by the influence of the remote ultra-violet band. Using this observed value of $n$, it is possible to calculate the wave-length $\lambda^{\prime \prime}$ of the ultra-violet band, assuming, as above, $m^{\prime \prime}=1 \cdot 13$.

This was found to be $\lambda^{\prime \prime}=18$, a value which looks very reasonable, judging from the photographs of the absorption in this region. It is of course impossible to determine experimentally the centre of this band, since the nitroso cuts off everything below $\lambda=20$. Whether or not a return of transparency would be found further along by employing fluorite plates and a vacuum spectrograph, it is impossible to say.

We are now in a position to calculate other values of $n$ in the ultra-violet and compare them with the observed values.

For $\lambda=36$ (the wave-length for which the lowest value of the refractive index was found experimentally), $n$ calcnlated by the first formula is $n=0.92$; by second formula, $n=1.08$; observed, $n=1.05$. For $\lambda=31$, by second formula $n=1.42$; observed, $n=1.43$.

Obviously we cannot apply the formula to that portion of 
the spectrum between wave-lengths 23 and 29 until we know the value of the extinction-coefficient in this region.

I feel certain that there are absorption-bands in the infrared, not only on account of the indications which the dispersion-formula gives, but also because when working with prisms of large angle 1 found that a fairly strong absorption occurred in the extreme visible red. It was not sufficient'y intense to make measurements impossible, and whether it was due to the presence of a weak diffused band similar to the one at $\lambda=25$, or to a strong band of metallic absorption, I was unable to determine, the observations being confined to the extreme edge. I expect to investigate this point as soon as our apparatus for the study of the infra-red region of the spectrum is in working order. I hope to be able in the near future to make a determination of the dielectric constant of the substance. With these points thoroughly investigated, a more rigorous application of the dispersion-formula to the results will be possible. I feel less hopeful of getting satisfactory data regarding the absorption, but shall at all events make the attempt.

\section{The Dispersion of Toluine.}

In examining the ultra-violet absorption of some organic solvents, I found that toluine has a fairly strong and narrow absorption-band at wave-length $\cdot 00027$, in addition to the band of metallic absorption below 00020 , which is the one which cliefly influences the dispersion of the substance. It occurred to me that it would be interesting to determine the dispersion of the substance through this region, and see to what extent the curve was modified by the weaker band. So far as I was able to find, the uitra-violet dispersion of tolnine has never been determined, which is not very surprising, since only very acute prisms transmit anything below the band above mentioned. The same method was employed as in the case of nitroso-dimethyl aniline, namely, the crossedprism method adapted to the quartz spectrograph. The dispersion was first measured in the visible spectrum with a hollow prism of $60^{\circ}$ angle. Small prisms were then made of quartz plates with the toluine between them, the angles varying from two to six degrees. The angles were determined from the photographs of the deviated spectra obtained with the quartz spectrograph, using the data obtained with the spectrometer in the blue region of the spectrum. Fig. 9 (PI. VIII.) shows one of the deviated spectra, the ultraviolet being to the left, and illustrates well the general form of the dispersion-curve in this region. Figs. 10 and 11 show the deviated and undeviated spectra, the one above the other, 
obtained with prisms of two different angles. It is at once apparent that the absorption-band at wave-length $\cdot 00027$ modifies the curve to no small degree. In the original negatives, a very slight trace of oppositely curved branches such as are always present, at the edges of strong absorption-bands is discernible. The band is quite narrow and fairly strong, yet it does not seem to modify the curve to any great degree on the red side, at least the curve has nothing like the steepness which it has in the remote ultra-violet. On the other side of the band, however, the curve is seen to be depressed to a considerable distance. The influence of the band seems therefore to be unsvmmetrical. This is to be ascribed to the fact, I believe, that the absorption on the ultra-violet side of this band is quite strong, as will be seen from tig. 11.

The plates were measured in the same manner as the nitroso plates, and a table of the refractive indices is given below.

\begin{tabular}{cl|cc}
$\lambda$. & $n$. & $\lambda$. & $n$. \\
2266 & 1.885 & 2681 & $1 \cdot 640$ \\
2288 & 1.8503 & 2750 & 1.628 \\
2314 & 1.821 & 2980 & $1 \cdot 595$ \\
2322 & $1 \cdot 808$ & 3250 & $1 \cdot 570$ \\
2330 & $1 \cdot 807$ & 3400 & 1.554 \\
2372 & $1 \cdot 767$ & 3659 & $1 \cdot 542$ \\
2470 & $1 \cdot 709$ & 3995 & $1 \cdot 526$ \\
2527 & $1 \cdot 679$ & 4799 & 1507 \\
2570 & 1.649 & &
\end{tabular}

The curve is shown graphically on Plate VI.

Applying the dispersion formula to the results, we find, neglecting the band at $\cdot 00027$ for the centre of the band of metallic absorption in the ultra-violet, the value $\cdot(000182$, which is not inconsistent with the photographic records. For the other constants we find $m=1.37$ and $m^{\prime}=\cdot 77$.

The sum of these two constants should give us the dielectric constant of the substance if no other bands of absorption are present.

The sum $m+m^{\prime}=2 \cdot 14$, while the dielectric constant is $2 \cdot 36$ (determination by Palaz), from which it seems probable that there is an absorption-band in the infra-red.

The formula expresses the dispersion very fairly except for a narrow range inmediately on the more refrangible side of the band at wave-length $\cdot 00027$. Adding another term to the formula will not help matters much in this region, in my opinion, owing to the indefinite nature of the absorption.

The substance seems to be worthy of a more complete investigation. 
Phil. Mag. Ser. 6, Vol. 6, Pl. VI.

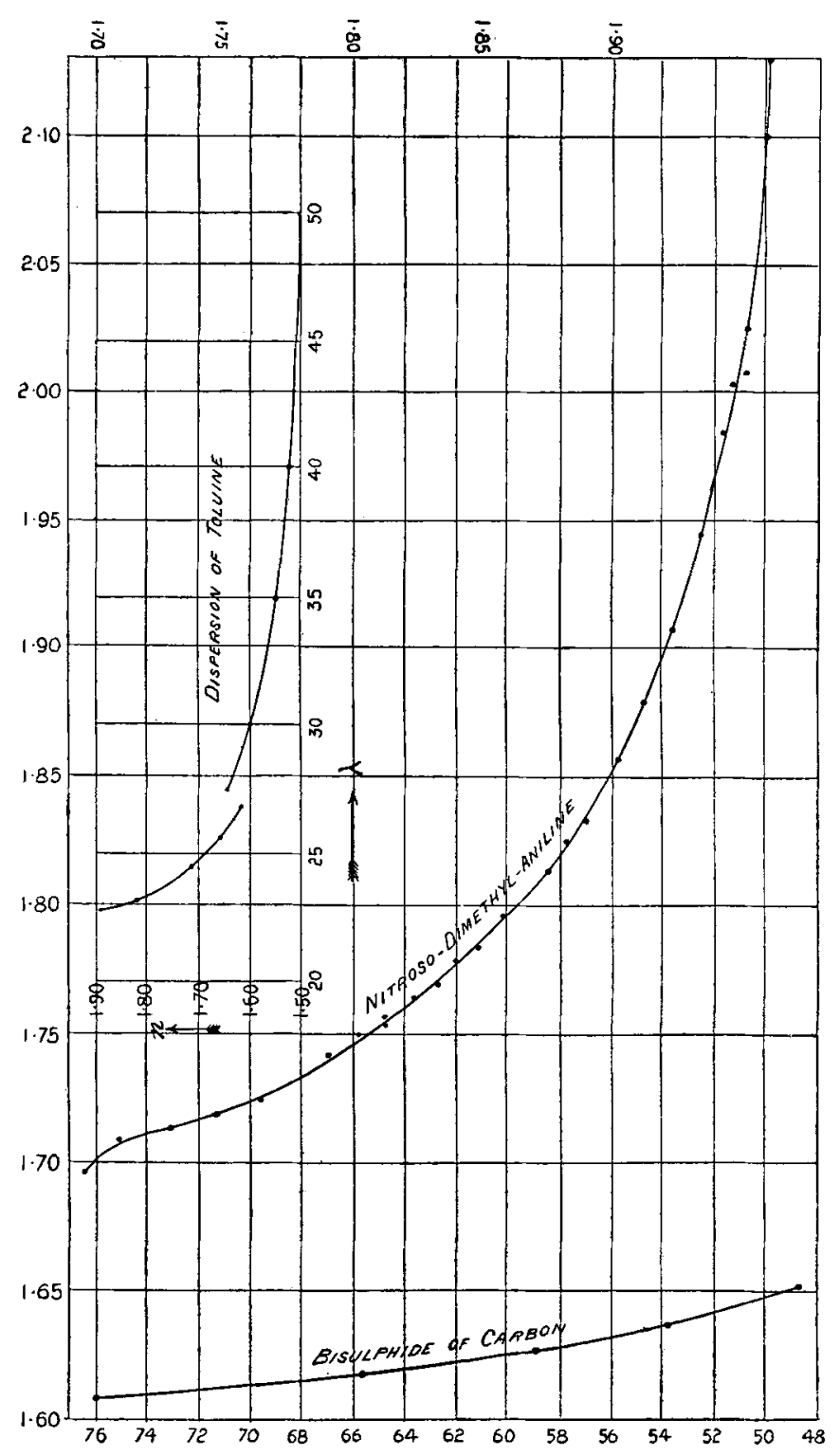


Phil. Mag. Ser. 6, Vol. 6, Pl. VII.

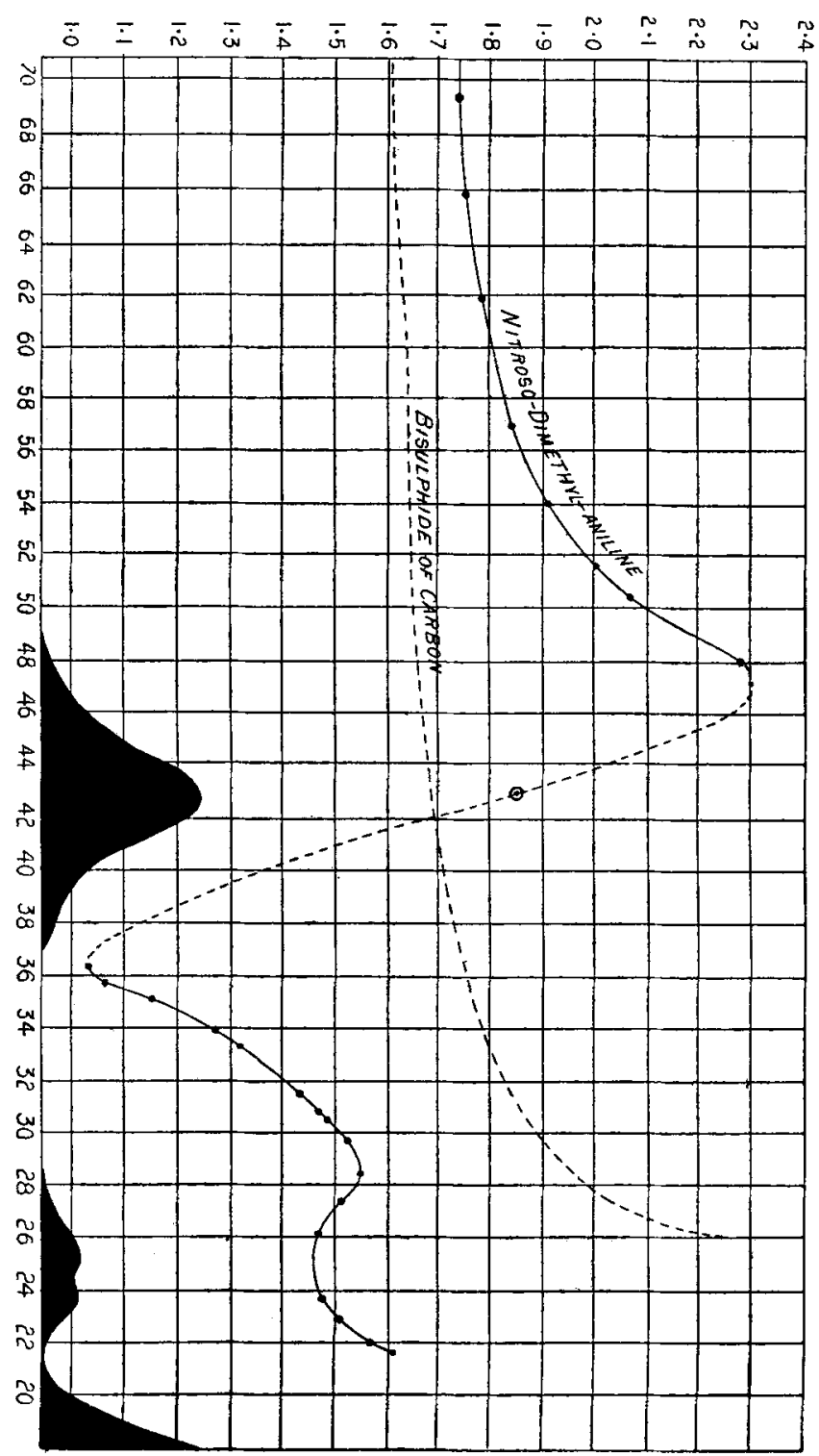


Phil. Mag. Ser. 6, Vol. 6, Pl. VIII.
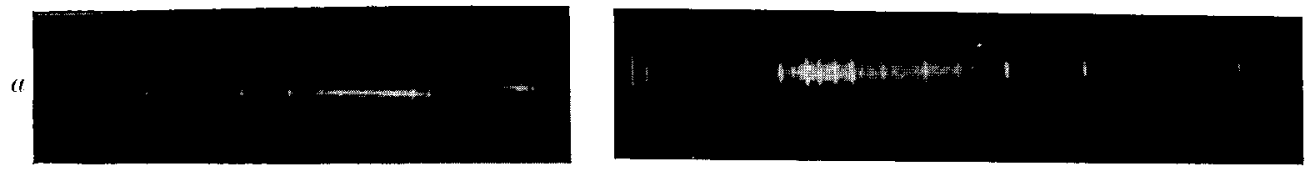

FIG. 7.
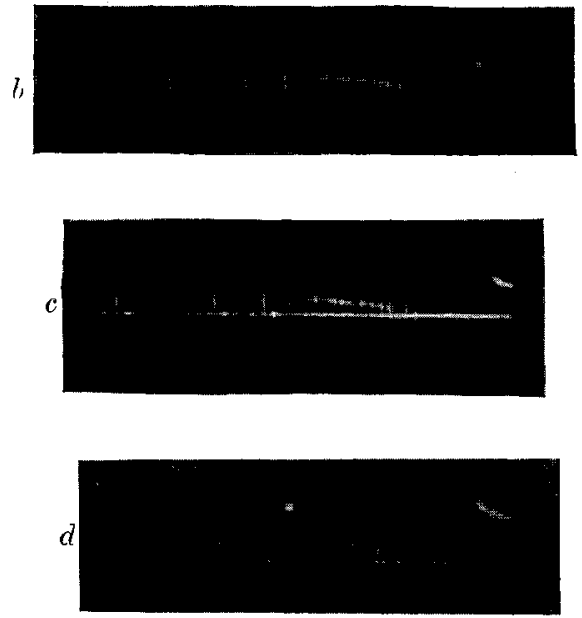

FIG. 6.

Fig. 8.

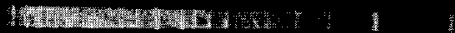

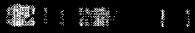

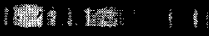

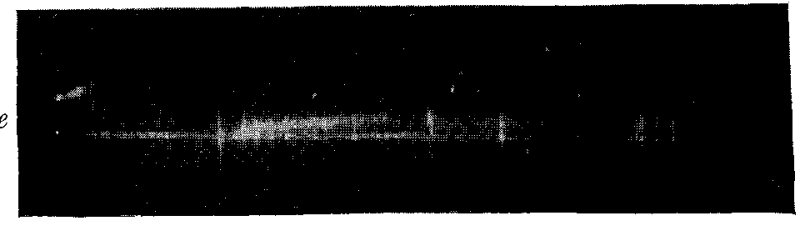

Fig. 3.

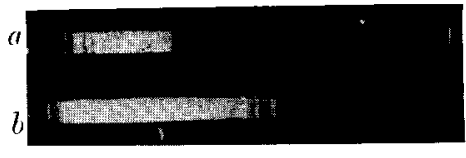

Fig. 5.

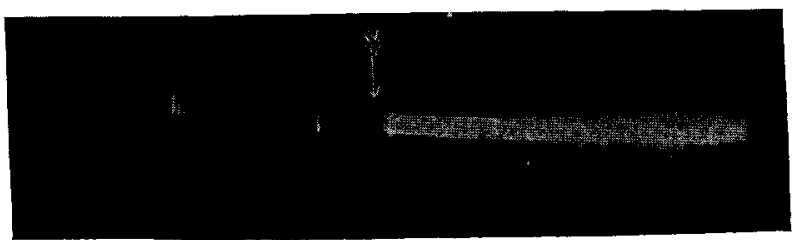

Fir. 9.

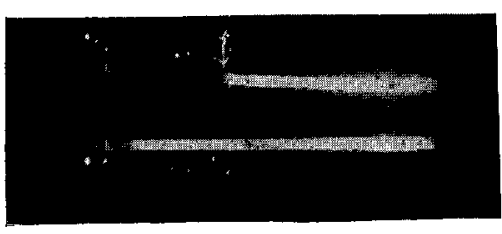

FIG. 10.

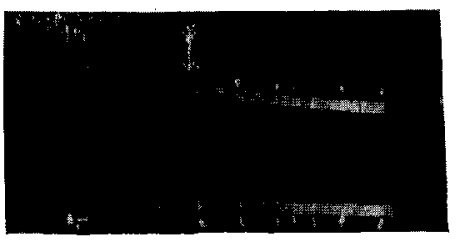

Fit. 11. 